\title{
Resistance and resilience of soil prokaryotic communities in response to prolonged drought in a tropical forest
}

\author{
Li Lingjuan 1, ${ }^{*}$, Preece Catherine ${ }^{1}$, Lin Qiang ${ }^{2}$, Brechet Laetitia ${ }^{1,3}$, Stahl Clément ${ }^{3}$, \\ Courtois Elodie $A^{4}$, Verbruggen Erik ${ }^{1}$
}

\begin{abstract}
${ }^{1}$ Research Group of Plants and Ecosystems (PLECO), Department of Biology, University of Antwerp, 2610 Wilrijk, Belgium

${ }^{2}$ Laboratory of Medical Microbiology, Vaccine \& Infectious Disease Institute, University of Antwerp, 2610 Wilrijk, Belgium

${ }^{3}$ UMR EcoFoG, CNRS, CIRAD, INRAE, AgroParisTech, Université des Antilles, Université de Guyane, 97310 Kourou, France

${ }^{4}$ Laboratoire Ecologie, Evolution, Interactions des Systèmes Amazoniens (LEEISA), Université de

Guyane, CNRS, IFREMER, Cayenne, French Guiana
\end{abstract}

* Corresponding author : Lingjuan Li, email address : Lingjuan.Li@uantwerpen.be

\begin{abstract}
:
Global climate changes such as prolonged duration and intensity of drought can lead to adverse ecological consequences in forests. Currently little is known about soil microbial community responses to such drought regimes in tropical forests. In this study, we examined the resistance and resilience of topsoil prokaryotic communities to a prolongation of the dry season in terms of diversity, community structure and co-occurrence patterns in a French Guianan tropical forest. Through excluding rainfall during and after the dry season, a simulated prolongation of the dry season by five months was compared to controls. Our results show that prokaryotic communities increasingly diverged from controls with the progression of rain exclusion. Furthermore, prolonged drought significantly affected microbial co-occurrence networks. However, both the composition and co-occurrence networks of soil prokaryotic communities immediately ceased to differ from controls when precipitation throughfall returned. This study thus suggests modest resistance but high resilience of microbial communities to a prolonged drought in tropical rainforest soils.
\end{abstract}

Keywords : drought, microbial communities, microbial network, tropical forest, resistance, resilience 


\section{Introduction}

Tropical forests act as an important carbon sink, and thus are pivotal to alleviating global climate change. The Amazon basin occupies a central position among global tropical forests as it represents half of all tropical vegetation biomass (Saatchi et al., 2011). However, it is under pressure from human activity and climate extremes (Findell et al., 2017). The Amazon forests are predicted to become warmer and drier in the coming decades (Bonal et al., 2016, Marengo et al., 2018). Intensified and prolonged seasonal droughts are projected to reduce ecosystem productivity, soil respiration and carbon uptake in the Amazon forests (Bonal et al., 2008, Aguilos et al., 2018, Bréchet et al., 2019). A more intense and longer dry season will also alter the distribution and structure of Amazon forest vegetation (Esquivel-Muelbert et al., 2019) and may affect microbial communities in as yet unknown ways. Because soil microbes play a dominant role in nutrient and carbon cycling, changes in their community diversity, structure or interactions (Faust \& Raes, 2012, Bardgett \& van der Putten, 2014) in response to drought may contribute to further positive global climate change feedbacks (Jansson \& Hofmockel, 2019).

Many studies have demonstrated that drought has considerable effects on soil microbial communities in drylands, temperate and Mediterranean ecosystems (Maestre et al., 2015, Kaisermann et al., 2017, Tóth et al., 2017, Guillot et al., 2019, Preece et al., 2019). This is thought to at least partly arise from inherent variation between taxa in their drought tolerance, such as that stemming from their cell-wall architecture, capacity for osmotic regulation, production of extracellular polymeric substances, spore formation, dormancy, and anatomical traits such as a unicellular versus a filamentous lifestyle (Schimel, 2018). Many of these traits are phylogenetically conserved at a relatively coarse level, and hence microbial responses to 
drought show a significant phylogenetic signal in a grassland soil (Amend et al., 2016). A general pattern that has emerged from prior research is that soil bacteria are relatively more sensitive to drought compared to fungi in terms of community structure (de Vries et al., 2018, Upton et al., 2018, Jansson \& Hofmockel, 2019). Among bacteria, Gram-positives such as Actinobacteria are generally favored under drought over Gram-negatives such as Proteobacteria (Sun et al., 2020). However, responses at finer phylogenetic levels also occur, as Bastida et al. (2017) found that the structure and diversity of bacterial communities were unaffected by drought at phylum and order levels, but were affected at genus level in the semiarid forest soil of Spain. Moreover, other than direct physiological responses, interactions among taxa may additionally attenuate or increase community responses of microbes, as suggested by pronounced changes in architectural properties of co-occurrence networks (de Vries et al., 2018).

The vast majority of studies on soil microbial communities in response to drought have been carried out in grasslands, temperate forests, or dryland ecosystems, which have an average annual precipitation ranging between around $300 \mathrm{~mm}$ and $1100 \mathrm{~mm}$ (Tóth et al., 2017, Guillot et al., 2019, Perez Castro et al., 2019). These studies generally indicate a high resilience of soil bacteria at a large ecosystem scale (Huang et al., 2020), and in one case, reach initial abundance three weeks after the end of the drought (Kaurin et al., 2018). Still, other studies indicate low resilience in for instance a grassland soil (Sheik et al., 2011), or dependence on prior drought exposure history which positively affected recovery in heathland soils (Barnard et al., 2013). Therefore, resilience may vary from one system to the other, and may depend on acclimation due to prior exposure, intensity and duration of drought (Piton et al., 2020). Amazonian tropical forests with a high mean annual precipitation are subjected to unusually intensive dry seasons in the current century (Aguilos et al., 2019), 
aligned with longer recovery times of plants to drought than in other ecosystems (Schwalm et al., 2017). Therefore, we may expect that soil microbial communities in Amazonian tropical forests to have lower resistance and resilience to drought compared to temperate ecosystems.

The few studies available for tropical forests confirm sensitivity to drought but display contrasted results on bacterial community composition from pronounced (Bouskill et al., 2013) to mixed or absent effects (Waring \& Hawkes, 2015, He et al., 2017). Moreover, to our knowledge, none followed up the community recovery process in detail. Still, they give clear indications of potential lasting effects: for instance, Waring \& Hawkes (2015) found that after an experimental drought in Costa Rica, soils exhibited increased respiration in situ due to a physiological shift in the microbial population. Bouskill et al. (2013) found after ten months of throughfall exclusion in Puerto Rico that the resulting change in bacterial phylogenetic community structure was not observed in soils that had experienced a prior drought event, suggesting a lasting change and an attenuated resistance to a subsequent drought. Therefore, more work is needed to ask whether tropical soil microbial communities fully recover from drought.

In the current study, we experimentally tested how prolonged drought impacts soil prokaryotic communities in a seasonal tropical forest in French Guiana. We investigated 1) whether a prolonged drought would increase the dissimilarity of prokaryotic communities from controls and change microbial co-occurrence network patterns, and 2) follow up whether the structure and co-occurrence of prokaryotic communities would recover in the months following a prolonged drought. We hypothesize that both resistance and resilience to prolonged drought are low, and that therefore the drought treatment leads to a lasting high 
dissimilarity of prokaryotic communities from control. Furthermore, we expect that the cooccurrence network changes upon prolonged drought, where taxa with a high resistance to drought become more connected leading the network as a whole to have higher connectivity.

\section{Materials and methods}

\section{Study site}

The study was conducted at the Paracou research station in French Guiana, South America $\left(05^{\circ} 16^{\prime} 54^{\prime \prime} \mathrm{N}, 52^{\circ} 54^{\prime} 44^{\prime \prime} \mathrm{W}\right)$. The decadal average annual rainfall at the study site was 3,102 $\mathrm{mm} \pm 70 \mathrm{~mm}$ and the average annual air temperature was $25.7 \pm 0.1{ }^{\circ} \mathrm{C}$ from 2004 to 2014 (Aguilos et al., 2019). The climate shows large seasonal variation in rainfall and is characterized by a wet season with heavy rains from December to July (precipitation between 300 and $500 \mathrm{~mm} \mathrm{mo}^{-1}$ ) and a dry season from mid-August to mid-November (precipitation < $100 \mathrm{~mm} \mathrm{mo}^{-1}$ ) (Aguilos et al., 2019). The soils in this site are mostly nutrient-poor acrisols (Bréchet et al., 2019).

\section{Experimental design}

In order to simulate a prolonged dry season, in five pairs of plots $(2.5 \times 2.5 \mathrm{~m})$ one plot each was covered by a translucent plastic tarpaulin $(3 \times 3 \mathrm{~m})$ to create a roof at $1.5 \mathrm{~m}$ above the soil surface reducing precipitation by $100 \%$. These plots served as "rain-exclusion", while the other plot within each pair served as uncovered "control". The tarpaulins were installed on October $9^{\text {th }}, 2017$ during the dry season and removed on April $27^{\text {th }}, 2018$ during the wet season. The imposed-drought effect on the soil by the tarpaulins was estimated from

September 2017 to July 2018 through soil volumetric water content (VWC) measurements 
taken with a dielectric soil moisture sensor, with general mineral soil calibration, at a depth of $5 \mathrm{~cm}$ (SM150T, Delta-T Devices, England). Simultaneously, soil surface temperature was recorded with a thermometer at a depth of $10 \mathrm{~cm}$ (HI 98501, Hanna instruments, USA). For further details on the experimental design see Bréchet et al. (2019).

\section{Sampling and sequencing}

Topsoil samples were collected before the roofs were installed (September 2017), and) monthly during the rain-exclusion period (with roofs) (January, February, March and April 2018) and after roof removal (May, June and July 2018) (Figure 1). Given that the top $2 \mathrm{~cm}$ of the soil is where the density of roots and particulate organic matter and by extension soil microbial activity are highest, we preferentially sampled that "assemblage" rather than taking a mix of assemblages that change with depth. Soil samples were taken using a tablespoon (1.5-cm diameter) to a depth of $0-2 \mathrm{~cm}$ excluding superficial litter at each sampling date. Three spoons of soil were collected within each plot and pooled to account for sampling effects/spatial heterogeneity. Composite soil samples were then sieved to $<2 \mathrm{~mm}$ diameter to remove small roots and stones and then stored in Eppendorf microcentrifuge tubes at $-20{ }^{\circ} \mathrm{C}$. All samples were lyophilized before shipment to Belgium for further microbial analyses. The analysis procedure of soil chemical and physical properties were presented in Bréchet et al. (2019). Soil samples were a sandy loam with a low nutrient concentration (17.29 g/ $/ \mathrm{kg}$ of C,

$1.18 \mathrm{~g} / \mathrm{kg}$ of $\mathrm{N}$, and $0.002 \mathrm{~g} / \mathrm{kg} \mathrm{P}$ ) and a low $\mathrm{pH}(3.84)$ (Table S1). Soil characteristics were not significantly/different between the rain-exclusion and control plots before roof installation (Table S1). 
Soil DNA was then extracted with the DNeasy PowerSoil Kit according to the manufacturer's instructions (Qiagen, Venlo, the Netherlands). The 16S rRNA gene was amplified from DNA with the universal primers 515F (5'-GTGCCAGCMGCCGCGGTAA3') and 806R (5'-GGACTACHVGGGTWTCTAAT-3') (Caporaso et al., 2011) targeting the prokaryotic V4 hypervariable region. In a first PCR, primers were amended with Illumina Nextera labels (Illumina Inc., San Diego, CA, USA). Each $25 \mu$ l reaction mixture contained 1 $\mu \mathrm{l}$ of the sample, $0.5 \mu \mathrm{M}$ of each forward and reverse primer, $1 \times$ PCR buffer, $200 \mu \mathrm{M}$ dNTPs and $1 \mathrm{U}$ Phusion High-Fidelity DNA polymerase. PCR conditions were as follows: initial denaturation at $98{ }^{\circ} \mathrm{C}$ for $60 \mathrm{~s}$, followed by 30 cycles of denaturation at $98{ }^{\circ} \mathrm{C}$ for $30 \mathrm{~s}$, annealing at $55{ }^{\circ} \mathrm{C}$ for $30 \mathrm{~s}$, extension at $72{ }^{\circ} \mathrm{C}$ for $30 \mathrm{~s}$, and then an additional extension of $72{ }^{\circ} \mathrm{C}$ for $10 \mathrm{~min}$. A second PCR was then performed using dual barcoded primers with Illumina adapters $(2.5 \mu \mathrm{l}$ of $50 \times$ diluted first PCR products template and $0.1 \mu \mathrm{M}$ of each primer). The conditions were $98{ }^{\circ} \mathrm{C}$ for $60 \mathrm{~s}$, followed by 12 cycles at $98{ }^{\circ} \mathrm{C}$ for $10 \mathrm{~s}, 63{ }^{\circ} \mathrm{C}$ for $30 \mathrm{~s}, 72{ }^{\circ} \mathrm{C}$ for $30 \mathrm{~s}$, and then $72{ }^{\circ} \mathrm{C}$ for $5 \mathrm{~min}$. PCR products were run on a $1.5 \%$ agarose gel to confirm successful PCR amplification. In total, 80 samples were then pooled into a single library and subjected to a gel extraction using a QIAquick Gel Extraction Kit (Qiagen). The library was quantified with quantitative PCR (Kapa Library Quantification Kits; Kapa Biosystems, Wilmington, MA, USA) and sequenced on the Illumina MiSeq platform (Illumina Inc.) using V3 chemistry with 300 cycles on both paired ends. The raw sequences are available at the European Nucleotide Archive under the accession number PRJEB43301.

\section{Data analyses}

Amplicon sequence analysis was performed using the USEARCH pipeline (Edgar, 2010).

Briefly, the paired-end reads were merged and primers were removed, and any sequences < 
200 bp removed. All reads were subsequently quality-filtered allowing for a maximum expected error of 0.5. Operational taxonomic units (OTUs) with $97 \%$ similarity were clustered using UPARSE (Edgar, 2013) after dereplication. One sample from control plots in February was discarded due to few reads, and the other samples were randomly rarified to the same number of reads $(11,981$ reads per sample) for all downstream analyses. The taxonomy assignment was performed with the RDP database (Cole et al., 2014) using the SINTAX algorithm (Edgar, 2016).

All statistical analyses were conducted in R software v.3.6.1 (Team, 2013). Alpha diyersity index in terms of Richness, Shannon-Wiener, Inverse Simpson and Pielou's evenness were calculated using the diversity function (in "vegan" package) (Oksanen, 2013). Initial exploration of the data showed a very strong block effect on community composition. Therefore, alpha diversity was analyzed using linear mixed effeets models (lme function in "nlme" package) (Pinheiro et al., 2017) with treatments and months as fixed factors, where block was a random factor. The analysis was done separately during the rain-exclusion period and after roof removal, respectively. The normality of residuals and homoscedasticity of the data were evaluated using the qqnorm and leveneTest function in "nlme" (Pinheiro et al., 2017) and "car" package (Fox et al., 2007). PERMANOVA was performed using the adonis function in "vegan" package on the data in different periods to evaluate the rain-exclusion, month and block effect, based on Bray-Curtis distances. Because block had a significant impact on microbial communities (Figure S1), we then further used partial distance-based redundancy analysis in the "vegan" package when evaluating the influence of prolonged drought on community composition, where block effect was partialled out. The statistical significance of axes and explanatory variables of partial dbRDA were tested with the anova function. For a visual representation of the treatment effect, the partial dbRDA axis 1 and 
axis 2 scores were used as proxies for community composition to evaluate the microbial community dissimilarity between rain-exclusion and control plots using Euclidean distance. Additionally, indicator species analysis was performed to identify potential indicator OTUs for rain-exclusion using multipatt function (IndVal metric) in the "indicspecies" package (De Caceres et al., 2016) where indicator value index was calculated by the 9,999-permutation test (De Cáceres et al., 2010). Only OTUs with a $p$ value $<0.001$ were selected as indicators. The visualization and clustering (based on pearson's correlation) of these indicator OTUs were conducted using aheatmap function in the "NMF" package (Gaujoux, 2014).

Co-occurrence patterns in microbial communities were assessed by performing network analyses using Random Matrix Theory (RMT)-based methods. This method identified the appropriate similarity threshold automatically for network construction (Deng et al., 2012). Four separate co-occurrence networks were constructed based on four groups of samples: those taken during the rain-exclusion period and those taken after roof removal, split by rainexclusion or control plots. To reduce rare OTUs in the dataset, the taxa with relative abundance $<0.1 \%$ were excluded for each network construction. A cutoff value (similarity threshold) for the similarity matrix was automatically generated using default settings. The cutoff values in this study were 0.72 and 0.74 during the rain-exclusion period and after roof removal, respectively, which serves to make the rain-exclusion network and control network comparable across periods. Network properties, including connectivity, average clustering coefficient, modularity and average path length were calculated. Connectivity, also termed as node degree, represents the connection strength of one node to other nodes. Modularity sérves as a measure of how well a network is divided into modules. Average clustering coefficient describes how well a node is connected to its neighbors, and average path length is the average distance between any two nodes (Deng et al., 2012). One hundred random 
networks with the same number of nodes and edges as the corresponding observed network were generated. Network construction and statistical analysis were performed using the Molecular Ecological Network Analyses Pipeline http://ieg2.ou.edu/MENA, for more details see Deng et al. (2012). Network visualizations were conducted using Gephi software (Bastian et al., 2009).

\section{Results}

\section{Changes in soil water content and temperature}

In September (dry season) before the roof was installed, VWC in the plots was about $11 \%$ (Figure 1). VWC of rain-exclusion plots was roughly maintained around $10 \%$ throughout the rain-exclusion period, whereas in control plots VWC followed precipitation events, resulting in significant increases from December (wet season start) onwards reaching a maximum value of $30 \%$ in February (Figure 1). After roof removal, VWC of rain-exclusion plots increased but remained significantly lower than control plots until July. The soil temperature only varied within a narrow range $\left(24^{\circ} \mathrm{C}-26^{\circ} \mathrm{C}\right)$ and was similar between rain-exclusion and control plots (Figure 1).

\section{Microbial community diversity and composition}

Microbial alpha diversity was unaffected by rain-exclusion, either during the rain-exclusion period or after roof removal (Table S2). Prolonged drought showed an impact on the microbial community composition (ANOVA, $p=0.002$ ), which itself varied from one sampling month to another $(p<0.001)$ indicating a clear temporal dynamic (Figure 2a, Table S1). Before the roof was installed, the microbial communities of rain-exclusion and control 
plots were not significantly different from each other $(p=0.204$, dissimilarity $=0.14$ (unitless partial dbRDA distance), Figure $2 b$, e). During the rain-exclusion period, microbial communities in the rain-exclusion and control plots diverged (Figure 2c, e), from a microbial dissimilarity of approximately 0.15 in September and January, reaching 0.27 in February and 0.38 in April (Figure 2e). After roof removal in May, the microbial communities of rainexclusion plots became similar to that of controls immediately and did not show a significant difference anymore with September levels $(p=0.387$, Figure $2 d$, e). The dissimilarities tended to slightly increase again in June $(0.18)$ and July $(0.21)$ but remained below those observed during rain-exclusion (Figure 2e).

At the phylum level, Proteobacteria $(47.59 \%)$ were dominant in all communities, followed by Actinobacteria (17.05\%) and Acidobacteria (9.57\%, Figure 3). The relative abundance of Actinobacteria in the rain-exclusion plots significantly increásed over time during the rainexclusion period, while it declined after roof removal (Figure 3). In line with this, we observed a slightly positive correlation between sample difference in relative abundance of Actinobacteria and that of VWC $\left(\mathrm{R}^{2}=0.28, p=0.012\right)$ during the rain-exclusion period (Figure S2). Other phyla showed little change during the rain-exclusion period. However, after the roofs were removed, Proteobacteria in the rain-exclusion plots sharply increased (in May), and even showed a higher relative abundance than in the control plots. Furthermore, we identified 35 individual indicator OTUs that responded strongly to drought, which were mainly representatives of Actinobacteria, Proteobacteria and Bacteroidetes (Figure 4). Most of indicator OTUS in the rain-exclusion were from Actinomycetales (Actinobacteria), with two ones from Dyella (Proteobacteria) and Rhizobiales (Proteobacteria). Specifically, these indicator OTUs from Actinomycetales were mainly affiliated with Streptomyces, Actinoallomurus, Rugosimonospora, Actinocatenispora and Kutzneria. Most of the indicator 
OTUs in the control were members of Burkholderiales (Proteobacteria), Betaproteobacteria (Proteobacteria), Cytophagales (Bacteroidetes), and Acidobacteria (Figure 4).

\section{Microbial co-occurrence patterns in response to precipitation change}

Co-occurrence networks of microbial communities were constructed to reveal the correlated response of microbial taxa to different treatments. Each of the four networks tended to be more clustered than expected by chance, as the average clustering coefficient and modularity values were significantly higher than those of random networks (Table 1).

During the rain-exclusion period, the rain-exclusion network had a similar number of nodes (OTUs) but more edges (links, $25 \%$ higher) than the control network (Table 1, Figure 5a). It also had a significantly higher $(p=0.026)$ node degree $(8.40)$ than the control $(6.46)$. The network density (0.09 versus 0.06$)$ and average clustering coefficient $(0.49$ versus $0.36, p=$ 0.002) were higher (Table 1) as well, with a higher occurrence frequency of Actinobacteria (39\% versus $23 \%$ ), but a lower occurrence frequency of Proteobacteria (34\% versus $45 \%$, Figure 5a) in the rain-exclusion than control network.

After roof removal, the rain-exclusion network had again a similar number of nodes and edges as the control network) (Table 1, Figure 5b). The average degree between the rainexclusion (7.03) and control networks (6.05) did not differ significantly $(p=0.162)$. Proteobacteria and Actinobacteria had similar frequencies in both the rain-exclusion and control network after roof removal (Figure 5b). 


\section{Discussion}

In this study, we asked whether a prolonged drought (a roughly doubling in the duration of the dry season) significantly alters the structure and composition of soil prokaryotic communities in a tropical rainforest in French Guiana, and whether they would recover upon return of normal rainfall in the months following the prolonged drought. Our results clearly indicate a pronounced change in the community composition and co-occurrence of soil prokaryotes. While drought is generally known to be an important driver of microbial community assembly in temperate soils (Bastida et al., 2017, Tóth et al., 2017), our results extend this to a mature tropical rainforest, where fluctuations in temperature and humidity are relatively mild and organisms may therefore lack adaptations to drought (Bonebrake \& Mastrandrea, 2010). However, we find that in comparison with findings in temperate systems (Sheik et al., 2011, de Vries et al., 2018), the microbial communities recovered surprisingly fast when precipitation throughfall returned. This suggests a significant resilience of the topsoil microbial community at this tropical forest site. In contrast to soil water content, the soil temperature only varied within a narrow range upon rain exclusion (increasing on average only by $0.16{ }^{\circ} \mathrm{C}$ ) during rain-exclusion period, and is therefore unlikely to have contributed to the altered community composition we report here.

Soil prokaryotic communities at this site were dominated by Proteobacteria and Actinobacteria, which agrees with most forest ecosystems (Chodak et al., 2015, Bu et al., 2018). Actinobacteria significantly increased in relative abundance as well as in prominence in the bacterial co-occurrence network when rain was excluded. This is in line with previous findings that members of this phylum are generally favored under drought in tropical forest soils (Bouskill et al., 2013) and in many temperate soils (Barnard et al., 2013, Perez Castro et 
al., 2019). Many Actinobacteria taxa, particularly members of the order Actinomycetales, resemble mycelial fungi in forming filaments which allow high access to resources in retreating water films (Bouskill et al., 2013). At the genus level, Streptomyces (order Actinomycetales) is the most dominant indicator and positively respond to drought in our study which is probably related to its great capacity to tolerant drought (Li et al., 2018). Furthermore, many Actinobacteria species have a high tolerance of osmotic stress (Bachar et al., 2010), and a high capability to continue to degrade recalcitrant carbon compounds under water limitation (Peltoniemi et al., 2012). These traits likely explain their superior performance in dry soils. Proteobacteria, another dominant phylum, were relatively unaffected by drought in our study. This is in line with a previous study in California grasslands (Barnard et al., 2013). In that study, it was suggested that Proteobacteria exhibit a resistant life-strategy with a reasonably stable relative abundance under drought and rewetting. However, some other studies showed the abundance of Proteobacteria was negatively correlated with drought, such as in a subtropical evergreen forest (Bu et al., 2018) and Mediterranean ecosystem (Perez Castro et al., 2019). The Proteobacteria were affected by drought at a lower taxonomic level in this study. Most Proteobacteria responded negatively to drought except Dyella which has also been previously reported to be drought resistant (Daffonchio \& Vigani, 2012), and Rhizobiales, which as a prominent bacterial order in rhizobial and lichen symbiosis, dominates in warm dry climates (Steidinger et al., 2019) or is highly desiccation tolerant (Erlacher et al., 2015), respectively. Those discrepancies at low taxonomic level lead Proteobacteria to be on average unaffected at phylum level.

Microorganisms are not isolated entities but interact with many other (micro-) organisms, with potential indirect effects and cascades. Our results demonstrate that prolonged drought impacts the network structure of microbial communities, in that co-occurrence networks of 
prokaryotes had greater connectivity (average degree) under drought. Connections between taxa were predominantly positive, which indicates that under drought there was a strong selection for taxa with similar environmental preferences and/or positive interspecific interactions (Blanchet et al., 2020, Lin et al., 2021). Because many of the taxa that become more connected were members of a presumably drought-tolerant phylum (Actinobacteria), it seems plausible that especially this shared trait is responsible for the positive co-occurrences, but positive interactions such as cross-feeding (Goldford et al., 2018) or exudation of osmolytes (Roberson \& Firestone, 1992) may contribute as well. The higher connectivity under drought found here is consistent with a previous finding in temperate grassland soil (de Vries et al., 2018), and may thus suggest community responses in this tropical soil respond analogously to drought. However, this result contrasts with a precipitation manipulation experiment in a Chinese seasonal subtropical forest, where prokaryotic networks were unaffected by an alternation of intensified drought and intensified precipitation (He et al., 2017). A potential reason for this discrepancy is that the drought intensity imposed here was more severe as we varied the duration and intensity of drought simultaneously, whereas in $\mathrm{He}$ et al. (2017) the duration was unaltered. Another potential reason is the difference in soil textures between the two studies. Acrisols such as studied here have a lower organic matter content leading to larger variation in soil water content compared to Ultisols such as in $\mathrm{He}$ et al. (2017). More work is needed to resolve the dependencies of microbial networks with respect to relevant drought scenarios in tropical forests, ideally inspired by realistic climate modeling forecasts.

Despite the significant drought-induced changes in i) microbial community structure, ii) relative abundance of particular taxa such as actinomycetes, and iii) co-occurrence network connectivity, we found prokaryotic communities recovered immediately after the onset of 
rain. This was both the case for community composition, and for network connectivity levels. The rewetting after a drought period not only releases soil microbes from water stress, but also creates a resource pulse that fuels soil microbial growth, which is called the "Birch effect" (Schimel, 2018). Indeed, in a previous report from the current study system, soil $\mathrm{CO}_{2}$ efflux increased as a result of rewetting after rain exclusion (Bréchet et al., 2019). Some microbes can take advantage of this pulse better than others, and there is an indication that Actinobacteria have a competitive disadvantage during rewetting (Barnard et al., 2013). This may account for the sharp decrease in the relative abundance of Actinobacteria, and the increase of others such as Proteobacteria in the rain-exclusion plots after roof remoyal. Many Proteobacteria have copiotrophic lifestyles analogous to $r$ - strategists and may thus rapidly respond to high nutrient availability (Fierer et al., 2007). Furthermore, the recovery we observed in this tropical forest is faster than reported in many studies in temperate soils, where multiple months were required for full or partial community recovery (Schwalm et al., 2017) and lasting changes in network architecture are observed (de Vries et al., 2018). Part of the explanation may lie in the generally high productivity of warm and moist tropical ecosystems, allowing communities to change at a faster pace than systems where the environment is more restrictive on microbial metabolic activity (Davidson \& Janssens, 2006). Another explanation may be that in our study only a limited area of soil was exposed to drought, whereas most vegetation was not: the area of the rain-exclusion plots would only affect a modest fraction of root systems of multiple mature trees. This potentially contrasts with the effect of a drought at a larger scale, which may have more severe effects on root activity and turnover, or even lead to whole tree mortality. These attributes may slow down the recoyery of microbial communities. 


\section{Conclusion}

This study elucidates the patterns of soil prokaryotic community response to, and recovery from, a prolonged drought in a French Guianan tropical forest. The similar response trajectories of the prokaryotic communities in terms of site-specific community composition and structure between this site and other temperate soils indicate that these life-strategies may be indicative of general patterns. Microbial co-occurrence subjected to drought probably indicates similar adaptions of interconnected taxa to drought. Furthermore, in contrast to many other studies, we find that prokaryotic communities recovered quickly once rainfall is no longer excluded, suggesting a high resilience. Future studies now need to evaluate whether this will continue to be the case in repeated drought with various intensity and/or duration, as this may have an important impact on the continued functioning of this vital ecosystem.

\section{Acknowledgments}

The authors acknowledge funding by the University of Antwerp BOF/GOA-project REGIME SHIFT. This work further benefited from an Investissements d'Avenir grant managed by Agence Nationale de la Recherche (CEBA, ref. ANR-10-LABX-25-01) and was supported by the European Research Council Synergy grant ERC-2013-SyG-610028-IMBALANCE-P. We are grateful to Thomas Saint-Germain, Jocelyn Cazal, Maricar Aguillos, Benoit Burban, Jean-Yves Goret, Baptiste Rabineau and the master students of the Module FTH for their help in the field. 


\section{References}

Aguilos M, Hérault B, Burban B, Wagner F \& Bonal D (2018) What drives long-term variations in carbon flux and balance in a tropical rainforest in French Guiana? Agric For Meteorol 253: 114-123. Aguilos M, Stahl C, Burban B, Hérault B, Courtois E, Coste S, Wagner F, Ziegler C, Takagi K \& Bonal D (2019) Interannual and Seasonal Variations in Ecosystem Transpiration and Water Use Efficiency in a Tropical Rainforest. Forests 10: 14.

Amend AS, Martiny AC, Allison SD, Berlemont R, Goulden ML, Lu Y, Treseder KK, Weihe C \& Martiny JB (2016) Microbial response to simulated global change is phylogenetically conserved and linked with functional potential. ISME J 10: 109-118.

Bachar A, Al-Ashhab A, Soares MI, Sklarz MY, Angel R, Ungar ED \& Gillor O (2010) Soil microbial abundance and diversity along a low precipitation gradient. Microb Ecol 60: 453-461.

Bardgett RD \& van der Putten WH (2014) Belowground biodiversity and ecosystem functioning. Nature 515: 505-511.

Barnard RL, Osborne CA \& Firestone MK (2013) Responses of soil bacterial and fungal communities to extreme desiccation and rewetting. ISME J 7: 2229-2241.

Bastian M, Heymann S \& Jacomy M (2009) Gephi: an open source software for exploring and manipulating networks. Vol. 3 p.^pp.

Bastida F, Torres IF, Andres-Abellan M, et al. (2017) Differential sensitivity of total and active soil microbial communities to drought and forest management. Glob Chang Biol 23: 4185-4203.

Blanchet FG, Cazelles K \& Gravel D (2020) Co-occurrence is not evidence of ecological interactions. Ecol Lett 23: 1050-1063.

Bonal D, Burban B, Stahl C, Wagner F \& Herault B (2016) The response of tropical rainforests to drought-lessons from recent research and future prospects. Ann For Sci 73: 27-44.

Bonal D, Bosc A, Ponton S, et al. (2008) Impact of severe dry season on net ecosystem exchange in the Neotropical rainforest of French Guiana. Glob Chang Biol 14: 1917-1933.

Bonebrake TC \& Mastrandrea MD (2010) Tolerance adaptation and precipitation changes complicate latitudinal patterns of climate change impacts. PNAS 107: 12581-12586.

Bouskill NJ, Lim HC, Borglin S, Salve R, Wood TE, Silver WL \& Brodie EL (2013) Pre-exposure to drought increases the resistance of tropical forest soil bacterial communities to extended drought. ISME J 7: 384-394.

Bréchet L, Courtois EA, Saint-Germain T, Janssens IA, Asensio D, Ramirez-Rojas I, Soong JL, Van Langenhove L, Verbruggen E \& Stahl C (2019) Disentangling Drought and Nutrient Effects on Soil Carbon Dioxide and Methane Fluxes in a Tropical Forest. Front Environ Sci 7.

Bu X, Gu X, Zhou X, Zhang M, Guo Z, Zhang J, Zhou X, Chen X \& Wang X (2018) Extreme drought slightly decreased soil labile organic $C$ and $N$ contents and altered microbial community structure in a subtropical evergreen forest. For Ecol Manag 429: 18-27.

Caporaso JG, Lauber CL, Walters WA, Berg-Lyons D, Lozupone CA, Turnbaugh PJ, Fierer N \& Knight R (2011) Global patterns of 16S rRNA diversity at a depth of millions of sequences per sample. PNAS 108: 4516-4522.

Chodak M, Golebiewski M, Morawska-Ploskonka J, Kuduk K \& Niklinska M (2015) Soil chemical properties affect the reaction of forest soil bacteria to drought and rewetting stress. Ann Microbiol 65: 1627-1637.

Cole JR, Wang Q, Fish JA, Chai B, McGarrell DM, Sun Y, Brown CT, Porras-Alfaro A, Kuske CR \& Tiedje IM (2014) Ribosomal Database Project: data and tools for high throughput rRNA analysis. Nucleic Acids Res 42: D633-D642.

Daffonchio DG \& Vigani G (2012) A drought resistance-promoting microbiome is selected by root system under desert farming. 
Davidson EA \& Janssens IA (2006) Temperature sensitivity of soil carbon decomposition and feedbacks to climate change. Nature 440: 165-173.

De Caceres M, Jansen F \& De Caceres MM (2016) Package 'indicspecies'. indicators 8: 1.

De Cáceres M, Legendre P \& Moretti M (2010) Improving indicator species analysis by combining groups of sites. Oikos 119: 1674-1684.

de Vries FT, Griffiths RI, Bailey M, et al. (2018) Soil bacterial networks are less stable under drought than fungal networks. Nat Commun 9: 3033.

Deng Y, Jiang Y-H, Yang Y, He Z, Luo F \& Zhou J (2012) Molecular ecological network analyses. BMC Biol 13: 113.

Edgar RC (2010) Search and clustering orders of magnitude faster than BLAST. Bioinformatics 26: 2460-2461.

Edgar RC (2013) UPARSE: highly accurate OTU sequences from microbial amplicon reads. Nat Methods 10: 996.

Edgar RC (2016) SINTAX: a simple non-Bayesian taxonomy classifier for $16 \mathrm{~S}$ and ITS sequences. bioRxiv 074161.

Erlacher A, Cernava T, Cardinale M, Soh J, Sensen CW, Grube M \& Berg G (2015) Rhizobiales as functional and endosymbiontic members in the lichen symbiosis of Lobaria pulmonaria L. Front Microbiol 6: 53.

Esquivel-Muelbert A \& Baker TR \& Dexter KG, et al. (2019) Compositional response of Amazon forests to climate change. Glob Chang Biol 25: 39-56.

Faust K \& Raes J (2012) Microbial interactions: from networks to models. Nat Rev Microbiol 10: 538550.

Fierer N, Bradford MA \& Jackson RB (2007) Toward an ecological classification of soil bacteria.

Ecology 88: 1354-1364.

Findell KL, Berg A, Gentine P, Krasting JP, Lintner BR, Malyshev S, Santanello JA, Jr. \& Shevliakova E (2017) The impact of anthropogenic land use and land cover change on regional climate extremes. Nat Commun 8: 989.

Fox J, Friendly GG, Graves S, Heiberger R, Monette G, Nilsson H, Ripley B, Weisberg S, Fox MJ \& Suggests M (2007) The car package. $R$ Foundation for Statistical Computing.

Gaujoux R (2014) An introduction to NMF package. Version 0206.

Goldford JE, Lu N, Bajić D, Estrela S, Tikhonov M, Sanchez-Gorostiaga A, Segrè D, Mehta P \& Sanchez A (2018) Emergent simplicity in microbial community assembly. Science 361: 469-474.

Guillot E, Hinsinger P, Dufour L, Roy J \& Bertrand I (2019) With or without trees: Resistance and resilience of soil microbial communities to drought and heat stress in a Mediterranean agroforestry system. Soil Biol Biochem 129: 122-135.

He D, Shen W, Eberwein J, Zhao Q, Ren L\& Wu QL (2017) Diversity and co-occurrence network of soil fungi are more responsive than those of bacteria to shifts in precipitation seasonality in a subtropical forest. Soil Biol Biochem 115: 499-510.

Huang L, Bai J, Wen X, Zhang G, Zhang C, Cui B \& Liu X (2020) Microbial resistance and resilience in response to environmental changes under the higher intensity of human activities than global average level. Glob Chang Biol 26: 2377-2389.

Jansson JK \& Hofmockel KS (2019) Soil microbiomes and climate change. Nat Rev Microbiol. Kaisermann A, de Vries FT, Griffiths RI \& Bardgett RD (2017) Legacy effects of drought on plant-soil feedbacks and plant-plant interactions. New Phytol 215: 1413-1424.

Kaurin A, Mihelič R, Kastelec D, Grčman H, Bru D, Philippot L \& Suhadolc M (2018) Resilience of bacteria, archaea, fungi and $\mathrm{N}$-cycling microbial guilds under plough and conservation tillage, to agricultural drought. Soil Biol Biochem 120: 233-245.

Li Y, Li Y, Wang LW \& Bao J (2018) Streptomyces dengpaensis sp. nov., an actinomycete isolated from desert soil. Int J Syst Evol Microbiol 68: 3322-3326. 
Lin Q, Dini-Andreote F, Meador TB, Angel R, Meszarosov L, Hedenec P, Li L, Baldrian P \& Frouz J (2021) Microbial phylogenetic relatedness links to distinct successional patterns of bacterial and fungal communities. bioRxiv.

Maestre FT, Delgado-Baquerizo M, Jeffries TC, et al. (2015) Increasing aridity reduces soil microbial diversity and abundance in global drylands. PNAS 112: 15684-15689.

Marengo JA, Souza CM, Thonicke K, Burton C, Halladay K, Betts RA, Alves LM \& Soares WR (2018) Changes in Climate and Land Use Over the Amazon Region: Current and Future Variability and Trends. Front Earth Sci 6: 228.

Oksanen J (2013) Vegan: ecological diversity. R Project 368.

Peltoniemi K, Straková P, Fritze H, Iráizoz PA, Pennanen T \& Laiho R (2012) How water-level drawdown modifies litter-decomposing fungal and actinobacterial communities in boreal peatlands. Soil Biol Biochem 51: 20-34.

Perez Castro S, Cleland EE, Wagner R, Sawad RA \& Lipson DA (2019) Soil microbial responses to drought and exotic plants shift carbon metabolism. ISME J 13: 1776-1787.

Pinheiro J, Bates D, DebRoy S, Sarkar D, Heisterkamp S, Van Willigen B \& Maintainer R (2017) Package ' $n l m e$ '. Linear and nonlinear mixed effects models, version 3.

Piton G, Foulquier A, Martinez - García LB, et al. (2020) Resistance-recovery trade - off of soil microbial communities under altered rain regimes: An experimental test across European agroecosystems. J Appl Ecol 58: 406-418.

Preece C, Verbruggen E, Liu L, Weedon JT \& Peñuelas J (2019) Effects of past and current drought on the composition and diversity of soil microbial communities. Soil Biol Biochem 131: 28-39.

Roberson EB \& Firestone MK (1992) Relationship between desiccation and exopolysaccharide production in a soil Pseudomonas sp. Appl Environ Microbiol 58: 1284-1291.

Saatchi SS, Harris NL, Brown S, et al. (2011) Benchmark map of forest carbon stocks in tropical regions across three continents. PNAS 108: 9899-9904.

Schimel JP (2018) Life in Dry Soils: Effects of Drought on Soil Microbial Communities and Processes.

Annu Rev Ecol Evol S 49: 409-432.

Schwalm CR, Anderegg WRL, Michalak AM, et al. (2017) Global patterns of drought recovery. Nature 548: 202-205.

Sheik CS, Beasley WH, Elshahed MS, Zhou X, Luo Y \& Krumholz LR (2011) Effect of warming and drought on grassland microbial communities. ISMEJ 5:1692-1700.

Steidinger BS, Crowther TW, Liang J, Van Nuland ME, Werner GD, Reich PB, Nabuurs G-J, de-Miguel S, Zhou M \& Picard N (2019) Climatic controls of decomposition drive the global biogeography of forest-tree symbioses. Nature 569: 404-408.

Sun Y, Chen HYH, Jin L, Wang C, Zhang R, Ruan H \& Yang J (2020) Drought stress induced increase of fungi:bacteria ratio in a poplar plantation. Catena 193: 104607.

Team RC (2013) R: A language and environment for statistical computing.

Tóth Z, Táncsics A, Kriszt B, Kröel-Dulay G, Ónodi G \& Hornung E (2017) Extreme effects of drought on composition of the soil bacterial community and decomposition of plant tissue. Eur J Soil Sci 68: 504-513.

Upton RN, Bach EM \& Hofmockel KS (2018) Belowground response of prairie restoration and resiliency to drought. Agric Ecosyst Environ 266: 122-132.

Waring BG \& Hawkes CV (2015) Short-term precipitation exclusion alters microbial responses to soil moisture in a wet tropical forest. Microb Ecol 69: 843-854. 


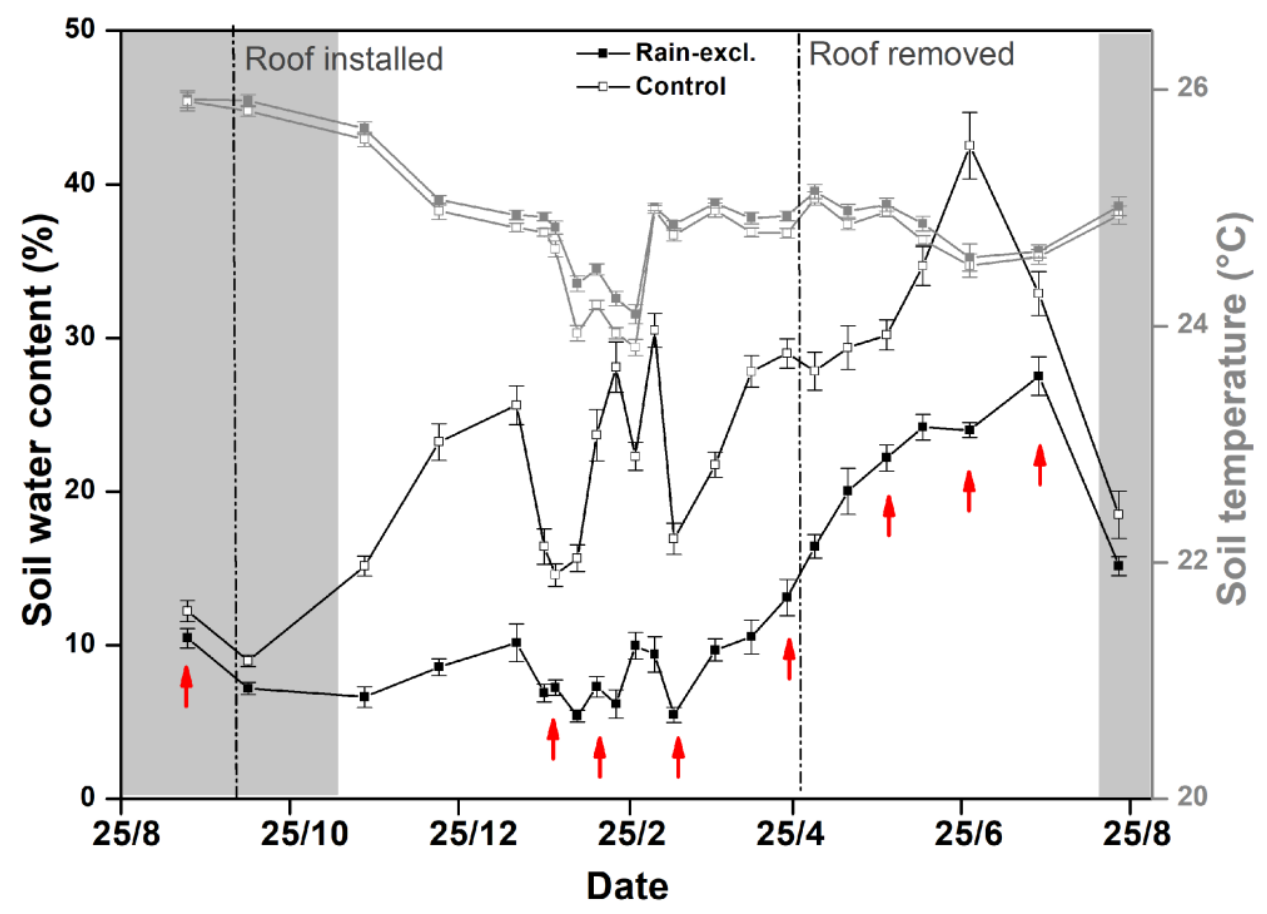

Figure 1. Temporal changes in mean topsoil volumetric water content (VWC, $5 \mathrm{~cm}$ depth) and topsoil temperature (mean $\pm \mathrm{SE} ; \mathrm{n}=5$ ) in the rain-exclusion (rain-excl.) and control plots over the experimental period in the Paracou tropical rainforest, French Guiana. The black lines indicate the values of VWC and the grey lines indicate the values of soil temperature. The grey shading represents the dry seasons (from mid-August to midNovember every year). Vertical dashed lines indicate the date of the roof installation (09/10/2017) and roof removal (27/04/2018) in the rain-exclusion plots. Red arrows indicate when soil samples were collected for microbial community analyses. 

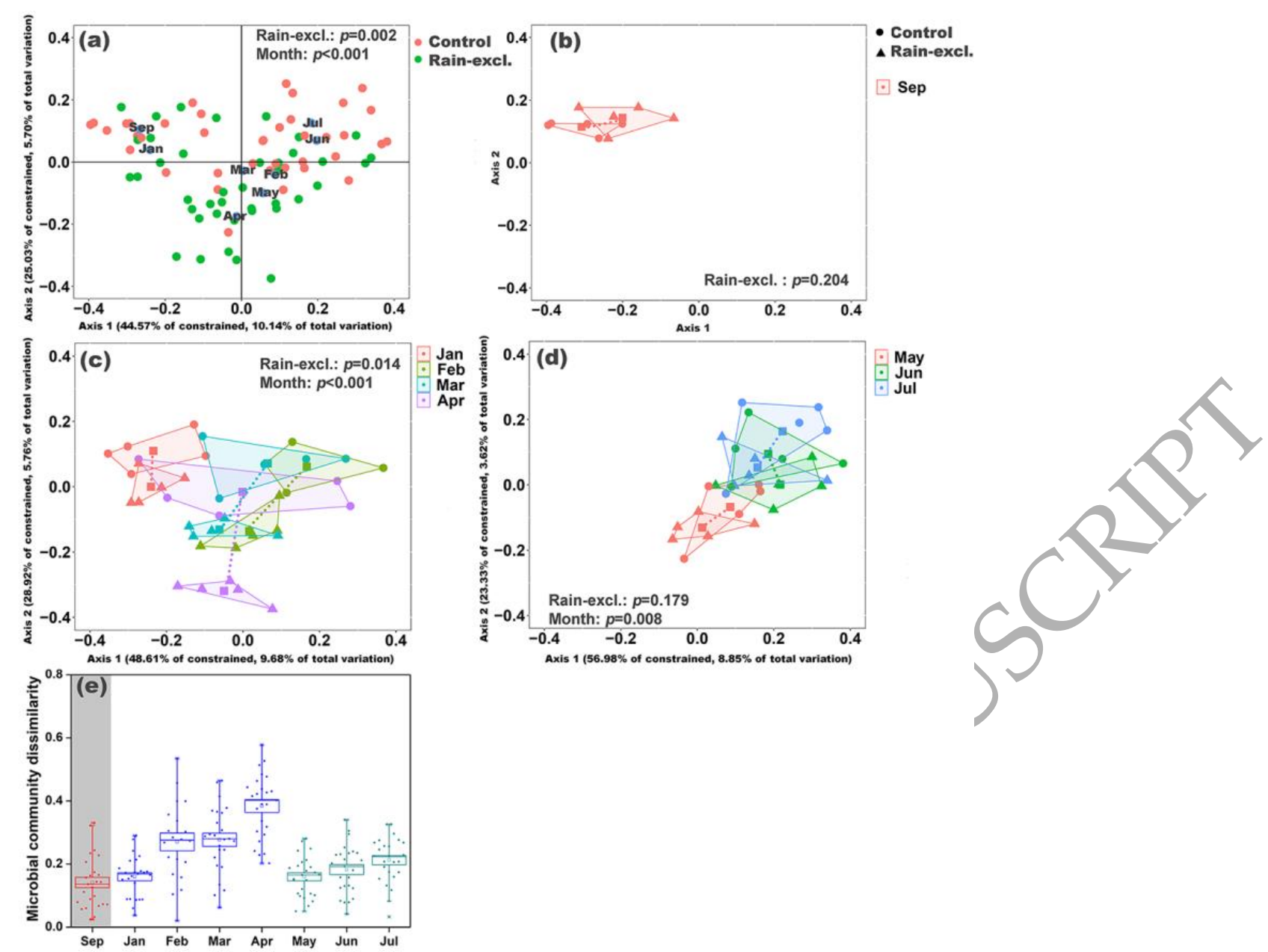

Figure 2. Partial distance-based redundancy analysis on the Bray-Curtis dissimilarity matrix in (a) all samples, (b) before roof installation (September), (c) during rain-exclusion period (January, February, March and April) and (d) after roof removal (May, June and July) in the Paracou tropical rainforest, French Guiana. The squares mean the centroids of each polygon and the dash lines mean the centroids connection between rain-exclusion and control plots in the same month. The values of $p$ are obtained through the anova function in the "vegan" package. The microbial dissimilarity between rain-exclusion and control plots within months are shown in (e), and the grey shading indicates the dry season. 

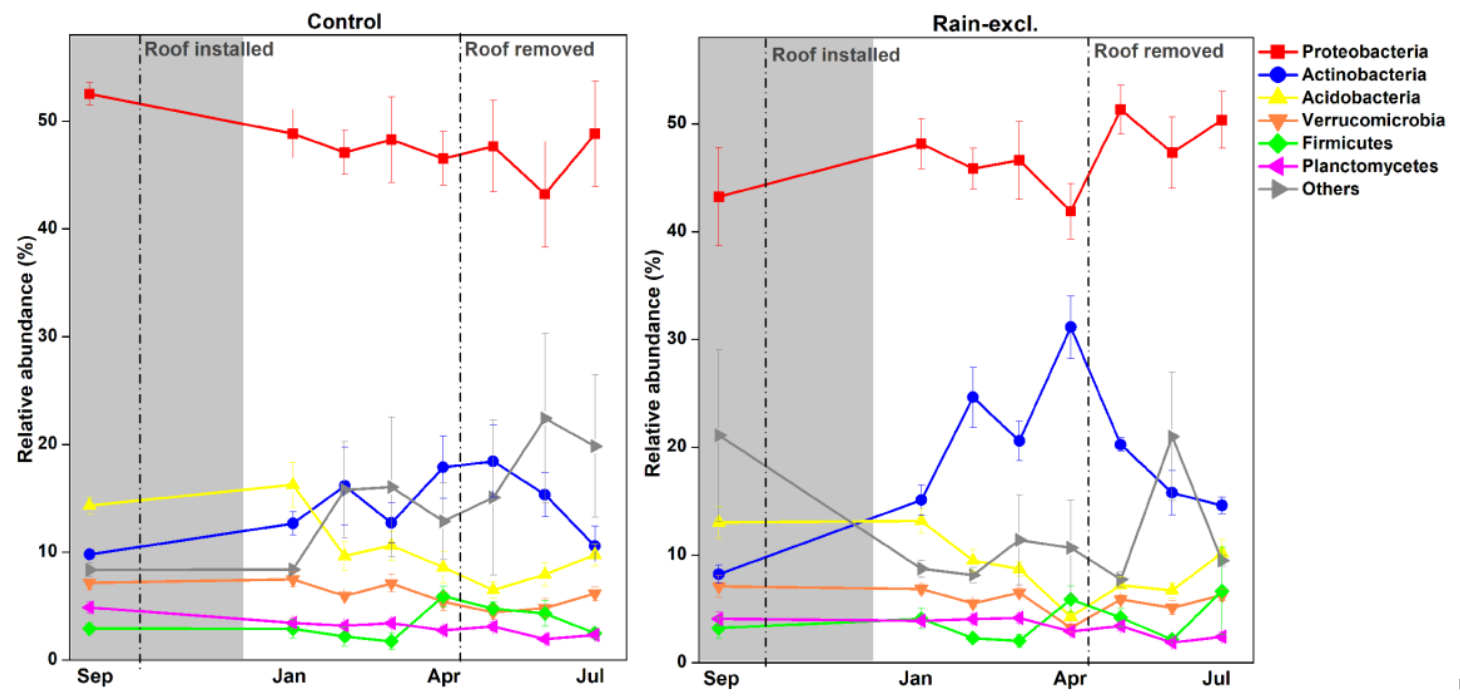

Figure 3. Relative abundance (mean $\pm \mathrm{SE}, \mathrm{n}=5$ ) at phylum level under different experimental conditions. The grey shading represents the dry season (from mid-August to mid-November). Vertical dashed lines indicate the date of the roof installation (09/10/2017) and roof removal (27/04/2018) in the rain-exclusion plots in the Paracou tropical rainforest, French Guiana. 


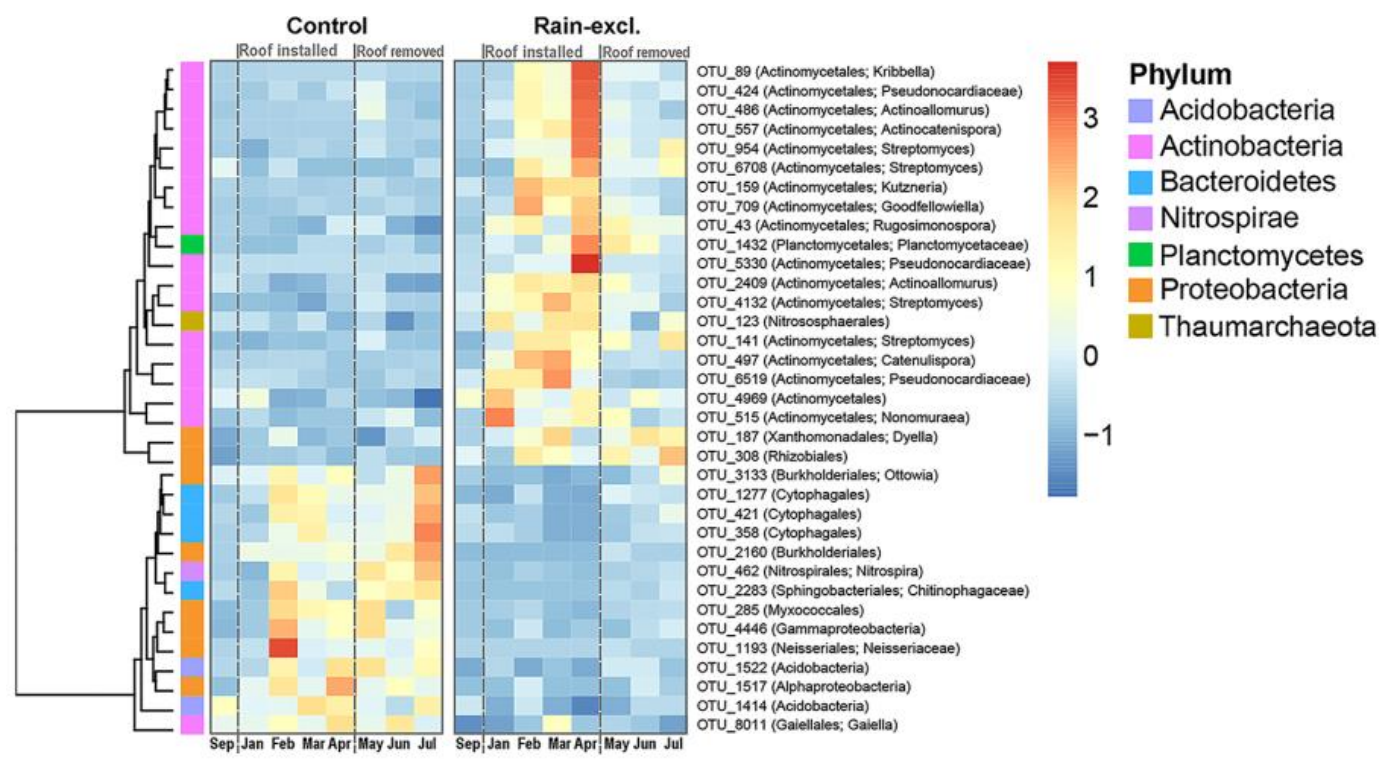

Figure 4. Heatmap of the relative abundance of microbial indicator OTUs from indicator species analysis associated with drought between rain-exclusion and control plots $(p<0.001)$. The row data for each OTU was $\mathrm{z}$-score transformed. The row annotations on the left side indicate the phylum of each OTU. Following OTU ID, the order name and the name of the next taxonomic level with the highest resolution available are written in brackets. Vertical dashed lines indicate the dates of roof installation (09/10/2017) and roof removal (27/04/2018) in the Paracou tropical rainforest, French Guiana. 


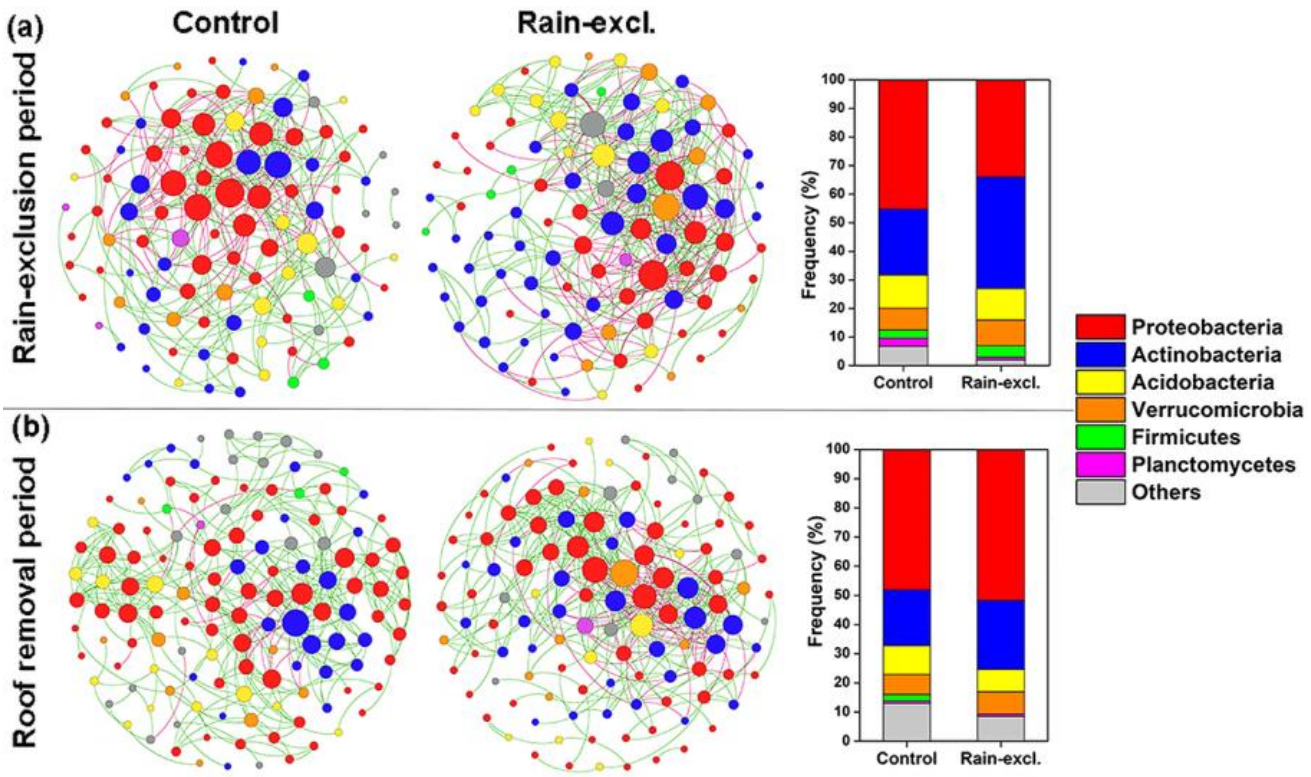

Figure 5. The co-occurrence network and frequency at the rain-exclusion and control plots (a) during rainexclusion period (January, February, March and April) and (b) after roof removal (May, June and July) in the Paracou tropical rainforest, French Guiana. Nodes represent individual OTUs colored by phylum and sized by connectivity (that is, node degree). Green links represent positive correlation and red links represent negative correlation. 
Table 1. Major properties of soil microbial networks under the rain-exclusion and control plots and associated random networks during the rain-exclusion period and after roof removal.

\begin{tabular}{|c|c|c|c|c|}
\hline & \multicolumn{2}{|c|}{ Rain-exclusion period } & \multicolumn{2}{|c|}{ After roof removal period } \\
\hline & Control & Rain-excl. & Control & Rain-excl. \\
\hline \multicolumn{5}{|l|}{ Empirical networks } \\
\hline Total nodes & 104 & 100 & 131 & 118 \\
\hline Total edges & 336 & 420 & 396 & 415 \\
\hline Average degree (avgK) & 6.46 & $8.40 *$ & 6.05 & 7.03 \\
\hline Average clustering coefficient (avgCC) & 0.36 & $0.49 *$ & 0.45 & 0.43 \\
\hline Density (D) & 0.06 & 0.09 & 0.05 & \\
\hline Modularity & 0.42 & 0.40 & 0.58 & \\
\hline \multicolumn{5}{|l|}{ Random networks } \\
\hline Average clustering coefficient (avgCC) & $0.13 \pm 0.02$ & $0.19 \pm 0.02$ & 0.0 & .02 \\
\hline Modularity & $0.31 \pm 0.01$ & $0.25 \pm 0.01$ & $0.35 \pm 0$. & $0.29 \pm 0.01$ \\
\hline
\end{tabular}

$*$ indicates significant difference $(t$ test, $p<0.05)$ between rain-exclusion and control networks. 